incomplete Burnett type coefficients of powers of $z^{-1}$ higher than the fourth. Above about $z=10$ it is also evident that for a computer carrying only twelve figures there is nothing to be gained in using a more elaborate converging factor than $R_{p} / u_{p}=0.5$.

National Research Council of Canada Ottawa, Canada

1. E. Dempsey \& G. C. Benson, "Tables of the modified Bessel functions of the second kind for particular types of argument," Can Jn. Phys., v. 38, 1960, p. 399. This paper contains tables of $K_{n}\left(\frac{\pi}{2} \sqrt{q}\right)$ for $q=1(1.0) 250$ and of $K_{n}\left(\frac{\pi}{3} \sqrt{q}\right)$ for $q=1(1.0) 300$. In both cases values for integral orders 0 to 10 were computed to ten significant figures.

2. R. B. DINGLE, "Asymptotic expansions and converging factors. I. General theory and basic converging factors" "Proc., Roy. Soc., London, v. 244A, 1958, p. 456.

3. R. B. DingLe, "Asymptotic expansions and converging factors. IV Confluent hypergeometric, parabolic cylinder, modified Bessel, and ordinary Bessel functions," Proc., Roy. Soc., London, v. 249A, 1959, p. 270.

4. D. BURNETT, "The remainders in the asymptotic expansions of certain Bessel functions," Proc., Camb. Phil. Soc., v. 26, 1930, p. 145. p. 138

5. E. JAhNKE \& F. EmDE, Tables of Functions, Fourth Edition, Dover, New York, 1945,

6. W. S. ALDIs, "Tables for the solution of the equation $\frac{d^{2} y}{d x^{2}}+\frac{1}{x} \cdot \frac{d y}{d x}-\left(1+\frac{n^{2}}{x^{2}}\right) y=0$," Proc., Roy. Soc., London, v. 64, 1899, p. 203.

\title{
On the Factors of Certain Mersenne Numbers
}

\section{By John Brillhart and G. D. Johnson}

1. Introduction. For the past 10 months the authors have been conducting a search for factors of certain Mersenne numbers on the IBM 701 at the Computer Center, University of California, Berkeley. The following is a report on the nature and results of that search.

2. Extent. Prime factors $q$ were sought for the numbers $M_{p}=2^{p}-1$ for primes $p<1200$ in the intervals indicated:

$$
\begin{array}{lrl}
p=101 & & 2^{30}<q<2^{35} \\
103 \leqq p \leqq 157, & p \neq 151 & 2^{30}<q<2^{31} \\
157<p \leqq 257 & & 1<q<2^{31} \\
257<p \leqq 1021, & p \neq 397 & 1<q<2^{30} \\
p=397 & & 1<q<2^{32} \\
1021<p<1200 & 1<q<2^{28}
\end{array}
$$

No factors $<2^{30}$ were examined for $101 \leqq p \leqq 157$, since these had already been investigated [1]. No $M_{p}$ were examined for $p<101$ or $p=151$, since these numbers have presumably been completely factored. Possible factors $<2^{35}$ were

Received May 5, 1960. 
also investigated for $M_{65537}$, the Mersenne number whose exponent is the "last" Fermat prime. $M_{397}$ was investigated to $2^{32}$ in the hope of finding more small factors.

\section{Results.}

A. Fifty-five new prime factors were discovered, 6 of which for $M_{p}$ below the traditional "limit" $p=257$. These factors are given in the accompanying table, and are indicated by *. Also included are all published prime factors, and 6 new ones (indicated by $\dagger$ ) of E. Karst, Brigham Young University. Thus, the table is believed to be a complete listing of all prime factors of $M_{p}$ for $p<1200$ known at this time. No factor was found for $M_{65637}$, whose character is still unknown. Since no factor was found to $M_{101}$ below its cube root, it is the product of two primes.

B. All known prime factors of $M_{n}, n<10000$, were tested and found correct, with the exception of the two misprints in $\mathrm{H}$. Riesel [2], as noted earlier by $\mathrm{J}$. Selfridge [3]. In addition, all factors were tested for multiplicity, but no new multiple factors appeared. Hence, to date, only a few multiple factors are known for composite exponents $n$, while none have been found for prime exponents, further supporting the conjecture that none exist.

\section{4. ,The Program.}

A. Structure. If $d \mid M_{p}$, then $d \equiv 1(\bmod 2 p)$. Also, since 2 is a quadratic residue of $M_{n}, n$ odd, then $d \equiv \pm 1(\bmod 8)$. Thus, the divisors, $d$, lie among the common terms $t_{n}$ of these arithmetic sequences.

In production these terms were generated consecutively by the repeated use of an increment table, which had also been constructed to produce no terms divisible by $3,5,7$, or 11 . (See [1].)

Divisibility of $M_{p}$ by each $t_{n}$ was tested by examining the remainder of $M_{p}$ $\left(\bmod t_{n}\right)$ for 0 .

For $101 \leqq p \leqq 223, M_{p}$ was reduced $\bmod t_{n}$ by multiple precision division.

Example 1. The remainder of $M_{101} \bmod t_{n}$ was computed for each $t_{n}$ by 3 divisions, until $t_{n}$ was $>2^{31}$, at which time an initial dividend of 67 binary places could be used. This change, which produced the remainder in only 2 divisions, was actually introduced when $t_{n}$ was $>2^{28}$ by using a modulus of $2^{\alpha} t_{n}, 0<\alpha \leqq 3$, instead of $t_{n}$, the error in the final remainder being removed after the last division by an appropriate number of subtractions of $t_{n}$, or multiples of $t_{n}$. This device was used consistently in all routines whenever possible.

When the program was first run for $p \geqq 223$, the final remainder was computed by residue methods consisting of successive squarings and doublings of the residue of some initial power of 2 , followed by a subtraction of 1 . Later it was realized, that in a double register machine like the 701, a residue between the initial and final residue could usually be multiplied by a power of 2 greater than the first without producing an illegal divide condition in the registers. The magnitude of the power that could be used was found to depend on the length of the registers (35 binary places) and the length of $t_{n}$.

This discovery decreased the testing time for each $t_{n}$ by about $30 \%$, but greatly complicated the programming, since from the many possible programs, one had to be chosen that required a minimum number of machine cycles. 
Table of Factors

\begin{tabular}{|c|c|c|c|}
\hline$p$ & Pactors & $p$ & Pactors \\
\hline 2 & 3 & 227 & \\
\hline 3 & 7 & 229 & $1504073 \cdot 20492753^{*}$ \\
\hline $\mathbf{5}$ & 31 & 233 & $1399 \cdot 135607 \cdot 622577$ \\
\hline 7 & 127 & 239 & $479 \cdot 1913 \cdot 5737 \cdot 176383 \cdot 134000609 *$ \\
\hline 11 & $23 \cdot 89$ & 241 & $22000409^{*}$ \\
\hline 13 & 8191 & 251 & $503 \cdot 54217$. \\
\hline 17 & 131071 & 257 & \\
\hline 19 & 524287 & 263 & 23671 . \\
\hline 23 & $47 \cdot 178481$ & 269 & $13822297^{*}$ \\
\hline 29 & $233 \cdot 1103 \cdot 2089$ & 271 & \\
\hline 31 & 2147483647 & 277 & 1121297 . \\
\hline 37 & $223 \cdot 616318177$ & 281 & 80929 . \\
\hline 41 & $13367 \cdot 164511353$ & 283 & 9623. \\
\hline 43 & $431 \cdot 9719 \cdot 2099863$ & 293 & \\
\hline 47 & $2351 \cdot 4513 \cdot 13264529$ & 307 & $14608903^{*} \cdot 85798519^{*}$ \\
\hline 53 & $6361 \cdot 69431 \cdot 20394401$ & 311 & 5344847 \\
\hline 59 & $179951 \cdot 3203431780337$ & 313 & $10960009^{*}$ \\
\hline 61 & 2305843009213693951 & 317 & 9511 . \\
\hline 67 & $193707721 \cdot 761838257287$ & 331 & \\
\hline 71 & $228479 \cdot 48544121 \cdot 212885833$ & 337 & $18199 \cdot 2806537 \dagger \cdot$ \\
\hline 73 & $439 \cdot 2298041 \cdot 9361973132609$ & 347 & \\
\hline 79 & $2687 \cdot 202029703 \cdot 1113491139767$ & 349 & \\
\hline 83 & $167 \cdot 57912614113275649087721$ & 353 & 931921 . \\
\hline 89 & 618970019642690137449562111 & 359 & $719 \cdot 855857 \cdot 778165529 *$ \\
\hline 97 & $11447 \cdot$ prime & 367 & $12479 \cdot 51791041^{*}$ \\
\hline 101 & & 373 & $25569151^{*}$ \\
\hline 103 & & 379 & \\
\hline 107 & prime & 383 & 1440847 . \\
\hline 109 & 745988807 . & 389 & $56478911^{*}$ \\
\hline 113 & $\begin{array}{l}3391 \cdot 23279 \cdot 65993 \cdot 1868569 \\
\cdot 1066818132868207\end{array}$ & 397 & $\begin{array}{c}2383 \cdot 6353 \cdot 50023 \cdot 53993 \\
\cdot 202471 \cdot 5877983 \dagger\end{array}$ \\
\hline 127 & prime & 401 & \\
\hline 131 & 263. & 409 & \\
\hline 137 & & 419 & 839 . \\
\hline 139 & & 421 & \\
\hline 149 & & 431 & $\begin{array}{l}863 \cdot 3449 \cdot 36238481^{*} \cdot 76859369^{*} \\
\quad .558062249^{*}\end{array}$ \\
\hline 151 & $18121 \cdot 55871 \cdot 165799 \cdot 2332951 \cdot$ prime & & \\
\hline 157 & 852133201 & 433 & \\
\hline 163 & $150287 \cdot 704161 \cdot 110211473^{*}$ & 439 & 104110607*. \\
\hline 167 & 2349023 & 443 & 887 . \\
\hline 173 & $730753 \cdot 1505447$. & 449 & 1256303 . \\
\hline 179 & $359 \cdot 1433$ & 457 & 150327409*. \\
\hline 181 & 43441 -1164193 · 7648337* & 461 & 2767 . \\
\hline 191 & 383 & 463 & $11113 \cdot 3407681 \dagger$. \\
\hline 193 & $13821503^{*}$. & 467 & $121606801^{*}$ \\
\hline 197 & 7487 & 479 & 33385343*. \\
\hline 199 & & 487 & 4871 . \\
\hline 211 & 15193 . & 491 & $983 \cdot 7707719 \dagger$. \\
\hline 223 & $18287 \cdot 196687 \cdot 1466449 \cdot 2916841$. & 499 & 20959 . \\
\hline
\end{tabular}


JOHN BRILLHART AND G. D. JOHNSON

TaBLE of FaCtors-Continued

\begin{tabular}{|c|c|c|c|}
\hline$p$ & Pactors & $p$ & Factors \\
\hline 503 & & 839 & 26849 . \\
\hline 509 & $12619129 \dagger \cdot$ & 853 & \\
\hline 521 & prime & 857 & 6857 \\
\hline 523 & & 859 & 7215601. \\
\hline 541 & & 863 & $8258911 \cdot 169382737^{*}$. \\
\hline 547 & 5471 . & 877 & $35081 \cdot 1436527 *$ \\
\hline 557 & $3343 \cdot 21993703^{*}$ & 881 & 26431 \\
\hline 563 & & 883 & $8831 \cdot 63577^{*}$ \\
\hline 569 & $15854617^{*} .55470673^{*}$ & 887 & $16173559^{*}$ \\
\hline 571 & $5711 \cdot 27409^{*}$ & 907 & 1170031 . \\
\hline 577 & 3463. & 911 & $1823 \cdot 26129303^{*}$ \\
\hline 587 & $554129 \cdot 2926783^{*}$. & 919 & \\
\hline 593 & 104369 & 929 & 13007 . \\
\hline 599 & & 937 & 28111 . \\
\hline 601 & $3607 \cdot 64863527^{*}$. & 941 & 7529 . \\
\hline 607 & prime & 947 & $295130657^{*}$. \\
\hline 613 & & 953 & 343081 \\
\hline 617 & 59233. & 967 & $23209 \cdot 549257^{*}$ \\
\hline 619 & 110183 . & 971 & \\
\hline 631 & & 977 & $867577 \cdot 1813313^{*}$ \\
\hline 641 & $35897 \cdot 49999^{*}$ & 983 & \\
\hline 643 & 3189281 & 991 & \\
\hline 647 & & 997 & \\
\hline 653 & $78557207^{*} \cdot 289837969^{*}$ & 1009 & 3454817 . \\
\hline 659 & 1319 & 1013 & 6079 . \\
\hline 661 & & 1019 & $2039 \cdot 75407^{*}$ \\
\hline 673 & $581163767^{*}$. & 1021 & $40841 \cdot 795808241^{*}$ \\
\hline 677 & & 1031 & $2063 \cdot 435502649^{*}$ \\
\hline 683 & 1367 . & 1033 & $196271 \cdot 36913223^{*}$ \\
\hline 691 & & 1039 & 5080711 \\
\hline 701 & $796337 \cdot 2983457^{*} \cdot 28812503^{*}$ & 1049 & $33569 \cdot 459463^{*}$. \\
\hline 709 & $216868921^{*}$ & 1051 & 3575503 . \\
\hline 719 & $1439 \cdot 772207^{*}$ & 1061 & \\
\hline 727 & & 1063 & \\
\hline 733 & & 1069 & \\
\hline 739 & & 1087 & $10722169^{*}$. \\
\hline 743 & 1487 . & 1091 & 87281 \\
\hline 751 & & 1093 & $43721 \cdot 111487^{*}$ \\
\hline 757 & $9815263 \cdot 561595591^{*}$. & 1097 & $980719 \cdot 4666639^{*}$ \\
\hline 761 & $4567 \cdot 6089^{*}$ & 1103 & 2207 \\
\hline 769 & & 1109 & \\
\hline 773 & $6864241 \cdot 9461521 \dagger$. & 1117 & 53617 . \\
\hline 787 & & 1123 & \\
\hline 797 & & 1129 & 33871 . \\
\hline 809 & & 1151 & \\
\hline 811 & 326023 & 1153 & 267497 . \\
\hline 821 & $419273207^{*}$ & 1163 & \\
\hline 823 & & 1171 & \\
\hline 827 & 66161 . & 1181 & 4742897 \\
\hline \multirow[t]{2}{*}{829} & $72953 \cdot$ & 1187 & $256393 \cdot 113603023^{*}$ \\
\hline & & 1193 & 121687 \\
\hline
\end{tabular}


In some cases, the initial residue was produced from a comparatively small power of 2 by a single division, while in others, it was obtained from a fairly large power of 2 by multiple-precision division.

Example 2. For $M_{397}, 4$ different programs were used, each improving on and replacing the preceding, when the length of $t_{n}$ permitted. The first divisor used was $t_{1}=3 \cdot 794+1=2383$, which also happens to be the first factor. This is shown below by the calculation schemes of the 4 programs, although only the first was actually used to test such a small possible divisor. With each scheme is also given the interval of $t_{n}$, for which it was used. The letters $i r$ after a residue indicate the initial residue used by the squaring part of the routine.

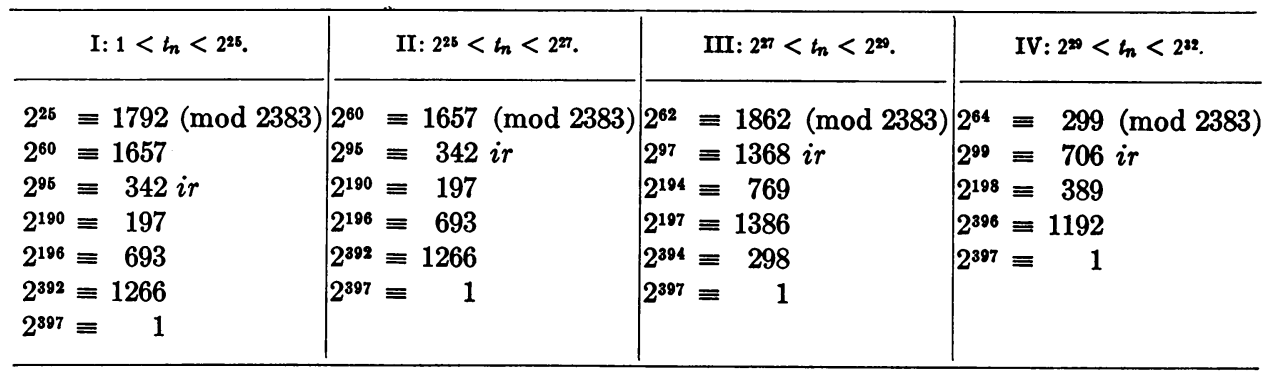

B. Production. The program was run for 60 hours, each $p<223$ requiring approximately 23 minutes, and each $p \geqq 223$ requiring from 8 to 18 minutes, the larger exponents taking progressively less time. The special number $M_{101}$ was run for 10 hours.

The routines used are believed to have been accurate, a fact which will be ascertained at a future time, when more rapid computers will accomplish in a few minutes, what has now taken many hours.

The authors would like to thank Professors R. M. Robinson and D. H. Lehmer for their suggestions and ideas, and also the staff of the Computer Center for their many courtesies.

University of California

Richmond, California

1. R. M. Robinson, "Some factorizations of numbers of the form $2^{n} \pm 1$, , MTAC, v. 11, 1957, p. 265-268.

p. $842-846$ , "Mersenne and Fermat numbers," Amer. Math. Soc. Proc., v. 5, 1954,

2. H. Riesel, "Mersenne numbers," MTAC, v. 12, 1958, p. 207-213.

3. J. L. Selfridat, Table Errata, MTAC, v. 13, 1959, p. 142. 